\title{
Towards single-molecule NMR detection and spectroscopy using single spins in diamond
}

\author{
V. S. Perunicic, ${ }^{*}$ L. T. Hall, D. A. Simpson, C. D. Hill, and L. C. L. Hollenberg \\ Centre for Quantum Computation and Communication Technology, School of Physics, University of Melbourne, Victoria 3010, Australia
}

(Received 25 December 2012; revised manuscript received 29 January 2014; published 27 February 2014)

\begin{abstract}
Nanomagnetometry using the nitrogen-vacancy (NV) center in diamond has attracted a great deal of interest due to its unique combination of room temperature operation, nanoscale resolution, and high sensitivity. One of the important goals for nanomagnetometry is to be able to detect nanoscale nuclear magnetic resonance (NMR) in individual molecules. Our theoretical analysis details a method by which a single molecule on the surface of diamond, with characteristic NMR frequencies, can be detected using a proximate NV center on a time scale of an order of seconds with nanometer precision. We perform spatiotemporal resolution optimization and subsequently outline paths to greater sensitivity. Our method is suitable for application in low and relatively inhomogeneous background magnetic fields in contrast to both conventional liquid and solid state NMR spectroscopy.
\end{abstract}

DOI: 10.1103/PhysRevB.89.054432

PACS number(s): 76.70.Hb, 75.75.Lf, 76.60.Lz

\section{INTRODUCTION}

Magnetic resonance (MR) based detection and imaging is an important tool across many areas of nanoscience. From a biomedical perspective, the need to better understand cellular processes at the nanoscale, occurring both naturally and as a result of introduced nanoparticles and/or molecular species, poses a significant and continuing challenge. The long history of magnetometry techniques in bioimaging, such as in electron spin resonance (ESR), nuclear magnetic resonance (NMR), and magnetic resonance imaging (MRI), have been successful in detecting the bulk properties of cells and their reactions $[1,2]$. However, all of these methods rely on detecting very large numbers of electronic or nuclear spins, and are hence fundamentally limited in their resolution. New methods such as magnetic resonance force microscopy (MRFM) are capable of detecting single electron or nuclear spins, yet require vacuous conditions at low temperatures $(<2 \mathrm{~K})$ [3-5].

In this work we theoretically describe how to perform NMR with the aim of detecting individual molecules under ambient conditions, using a single electronic spin as a NMR probe. The MR detector we consider is the electronic spin system of the nitrogen-vacancy (NV) center in diamond, which exhibits a number of fortuitous properties (for a review of the NV center see Ref. [6]) making it a very promising sensor: it is biocompatible, exhibits sustained fluorescence over arbitrarily long time scales, and is inherently a nanoscale magnetic sensor with high sensitivity. The NV center is effectively an electronic spin-1 system, whose ground state can be optically initialized and read out [7-11]. The coherence time of a NV spin several nanometers below a high purity diamond surface is relatively long at room temperatures, extending into millisecond time scales [12]. NV centers have been used as fluorescent biomarkers $[13,14]$, whose spin state can be coherently controlled inside living cells [15]. Applications span numerous fields including quantum computing, encryption [16-18], sources of single photons [19], and magnetic field sensing in the contexts of static (dc) [20] and oscillating (ac) [21] fields using single NV centers or ensembles [22]. For randomly fluctuating (FC) fields, particularly those encountered in biology, schemes

\footnotetext{
*vpe@unimelb.edu.au
}

for decoherence based magnetometry have been proposed [23-26]. Interest in electronic spin detection using NV centers is rapidly growing [27-31], while in biological contexts the detection of near individual atomic spin labels in an artificial cell membrane using the NV center as a probe has been demonstrated [32]. Ferritin molecules have been detected by virtue of their large electronic spin signal [33]. Recent landmark experiments demonstrate that the NV center is capable of identifying the presence of a relatively small number of nuclear spins, compared with bulk methods, external to the diamond lattice both by passive observation [34] and by pulsed manipulation of the target spins [35]. At present, methods to detect nonspecific single and paired nuclear spins within the diamond lattice are being developed [36,37], and methods for external molecule detection using the NV center based on detecting the natural flip-flop dynamics of nuclear dimer spins [38] or matching the Rabi frequency of the NV center with the energy levels of a target spin [39] have been recently proposed. An important goal that we investigate here is to use the NV center as a nanoscale magnetometer for the task of selective single-molecule NMR detection. Our approach is to detect the magnetization signal of an externally continuously driven spin in the target molecule, from which we determine the anisotropic NMR spectrum, resolving information about its chemical environment. As such, our method intrinsically allows for selective molecular detection.

\section{METHOD}

In this section we discuss the methodology by which the NV center in diamond can be used to detect a NMR spectrum of an external molecule; and where our method resides with respect to established liquid and solid state NMR spectroscopic techniques.

\section{A. Concept}

Our method is depicted schematically in Figs. 1 and 2. The application of a continuous resonant radio frequency (rf) field to the target spin drives the periodic transitions between its magnetic sublevels. This produces a magnetization signal that may be detected by the NV spin [Fig. 2(a)]. By applying pulse-based resonant microwave control to the NV spin (such 


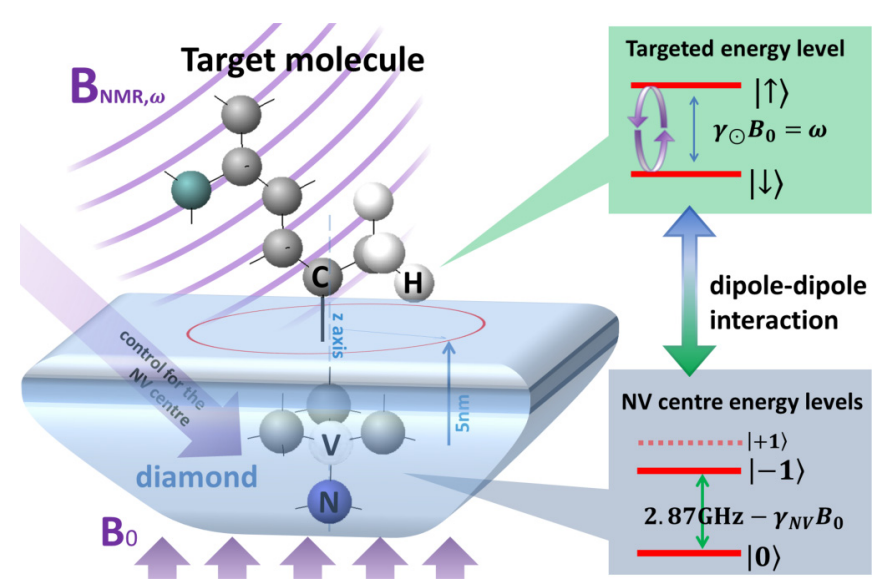

FIG. 1. (Color online) General schematic representation of the system containing an NV center and some single target molecule. The NV center is located in a diamond lattice approximately $5 \mathrm{~nm}$ under the surface. For simplicity, the surface corresponds to a (l 11 1) crystal plane, allowing the NV center quantization axis (z) to be perpendicular to the diamond surface. The microwave control is set to address only two of the center's three energy states and the NV center experiences a dipole-dipole interaction with the target nuclear spin. The target is subjected to an oscillating magnetic field $B_{\mathrm{NMR}}$ resonant with its (known) energy level separation, with the background magnetic field $B_{0}$ aligned along the $\mathrm{NV}$ center axis.

as spin-echo, CPMG, or Uhrig dynamic decoupling [40-42], for example) we may simultaneously increase its coherence time [43-46], thereby maximizing its response to the target signal. The frequency of the rf field may be chosen to match a particular transition frequency within the molecule, thereby allowing the method to be selective to a particular chemical species. In the examples illustrating the method the orientation of the NV center quantization axis was, without loss of generality, chosen to be perpendicular relative to the diamond surface, with the background magnetic field $B_{0}$ aligned along the quantization axis of the NV center, as depicted in Fig. 1, additional details are given in Sec. III A.

Continuous application of the driving rf magnetic field is a fundamental feature of our method. It provides a significantly higher spectral resolution compared to passive and pulsed techniques. Passive observation is based on using a NV spin control sequence as a filter function, thus the main limiting bound to their spectral resolution is the longitudinal coherence time $\left(T_{2 \mathrm{NV}}\right)$ of the $\mathrm{NV}$ center. The limiting factor of approaches based on nuclear spin pulses is power broadening as they require strong if fields in order to make the pulses relatively short compared to total observation time. As the continuous driving fields are applied throughout the full length of the observation, considerably weak rf fields are used, allowing for increased spectral resolution, while the effects of the NV coherence time are reduced to limiting the signal contrast without imposing a fundamental limit on the spectral resolution.

Initially, the $\mathrm{NV}$ probe can be characterized in the absence of a driving field $\left(B_{\mathrm{NMR}}=0\right)$, reflecting the influences of the entire environment to which it is exposed. The system is then analyzed while the target molecule is continuously exposed to (a)
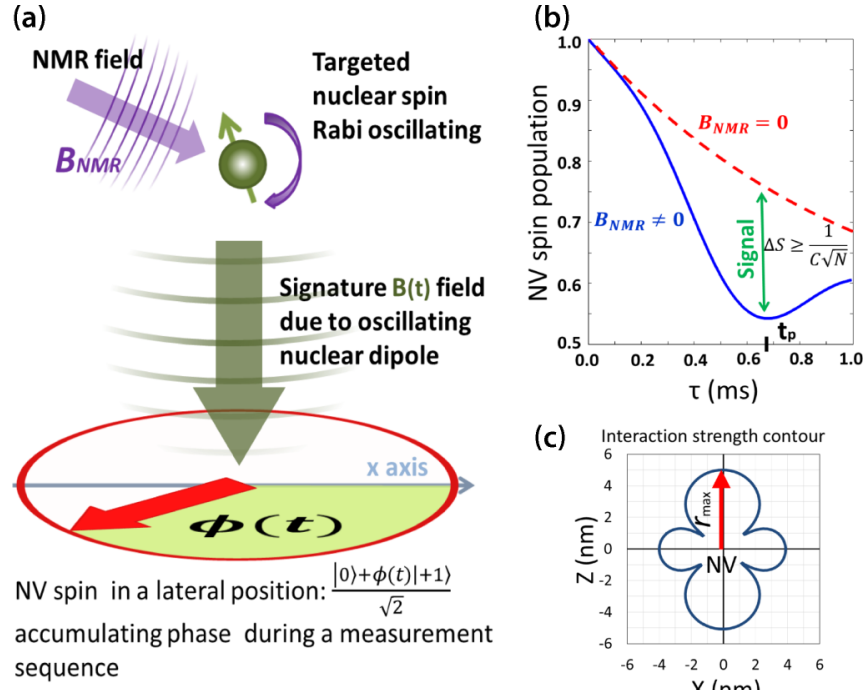

(c)

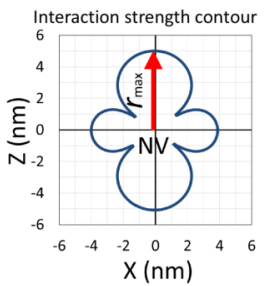

FIG. 2. (Color online) Measurement protocol. (a) The resonant rf field $B_{\mathrm{NMR}}$ induces oscillations in the specific targeted nuclear spin transition. The dipole of a targeted nucleus creates a fluctuating field $B(t)$, inducing a phase $\phi(t)$ in the NV spin which is transformed into change in the spin state population $\Delta S$ (signal). (b) The spin population of the NV center under a spin-echo sequence in the proximity $(5 \mathrm{~nm})$ of a single-molecule proton spin with (dashed, red line) and without (solid, blue line) the rf field. The signal is nonzero when the rf field is resonant to a targeted NMR transition. (c) The cross section of the interaction strength $k(\mathbf{r})$ contour between the targeted nuclear spin and the NV center. The maximum distance between the NV and the target $r_{\max }$ is defined as an intersection of the contour and the $z$ axis.

the rf field $\left(B_{\mathrm{NMR}} \neq 0\right)$, as illustrated in Fig. 2(b). We define the change in the NV spin population as the signal $(\Delta S)$, by comparing the outcomes of those two cases.

To provide a concrete example of how this technique may be implemented, we consider the following target species: aldehyde $(-\mathrm{CHO})$, hydroxymethyl $\left(-\mathrm{CH}_{2} \mathrm{OH}\right)$, and methyl $\left(-\mathrm{CH}_{3}\right)$ groups attached to the diamond surface. The aldehyde group exemplifies the detection of only one external proton spin, while the other two examples represent detection of two and three proximate protons. The NV center is positioned several nanometers below an individual target, as depicted in Fig. 3. We are primarily interested in obtaining the NMR spectrum of each individual group and using that information to detect the presence of a targeted molecule attached to the diamond surface. The Hamiltonian of the combined system is given by

$$
\begin{aligned}
H_{\mathrm{system}} & =H_{\mathrm{NV}}+H_{\odot}+H_{\mathrm{d}-\mathrm{d}}, \\
H_{\mathrm{NV}} & =\hbar \gamma_{\mathrm{NV}} B_{0} Z_{\mathrm{NV}}+\Delta Z_{\mathrm{NV}}^{2}+\Pi(t), \\
H_{\odot} & =\sum_{i} \hbar \gamma_{\odot}^{i}\left\{B_{0} Z_{\odot}^{i}+B_{\mathrm{NMR}}\left[X_{\odot}^{i} \cos (\omega t)+Y_{\odot}^{i} \sin (\omega t)\right]\right\}, \\
H_{\mathrm{d}-\mathrm{d}} & =K_{\mathrm{NV} \odot}+K_{\odot \odot},
\end{aligned}
$$

where $H_{\mathrm{NV}}$ encompasses the NV center's zero field splitting $\Delta=2.87 \mathrm{GHz}$, its interaction with the background $B_{0}$ and NVcontrol fields $\Pi(t) ; H_{\odot}$ is the interaction of each target nuclear spin $i$ with the background field and the rf field of frequency $\omega$; 
Molecular target
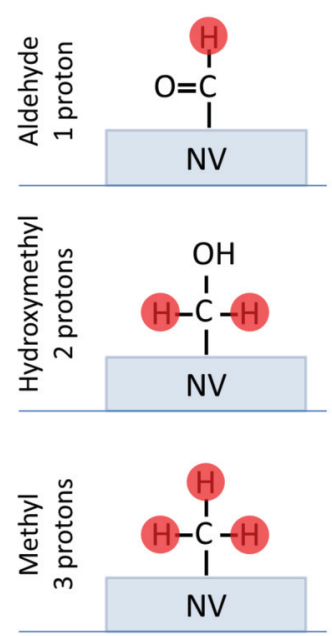

Sweep over target driving frequency
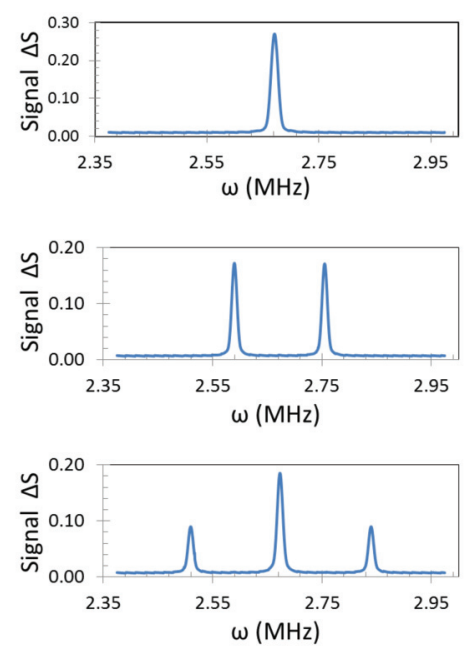

FIG. 3. (Color online) The NMR spectrum of protons in three chemical species: aldehyde, hydroxymethyl, and methyl groups, obtained using the NV center. The groups are attached to the surface of the diamond at $r_{\max }=5 \mathrm{~nm}$ above the NV center. The spectra of the highlighted protons is depicted in the adjacent plots (right). The hydroxyl proton of the hydroxymethyl group is inactive, depicting proton exchange in an aqueous solution. Equivalently, the rest of the diamond surface can also be terminated in with NMR neutral groups [55,56] (e.g., oxygen, hydroxyl, carboxyl). Note, for clarity the diamond surface was chosen such that both the background magnetic field and the NV center quantization axis are perpendicular to the diamond surface.

$X_{\mathrm{NV} / \odot}, Y_{\mathrm{NV} / \odot}$, and $Z_{\mathrm{NV} / \odot}$ are the Pauli spin matrices of the $\mathrm{NV}$ center or the target spin, respectively, $\mathbf{S}_{\mathrm{NV} / \odot}$ is a vector of Pauli matrices, and $\boldsymbol{r}$ is a vector between the NV center and a target nucleus or between the target nuclei themselves. The total dipole interaction term $H_{\mathrm{d}-\mathrm{d}}$ contains the dipole interactions between the NV spin and each of the target nuclear spins $K_{\mathrm{NV} \odot}$ as well as the interaction between target nuclei themselves $K_{\odot \odot}$, given by

$$
\begin{aligned}
K_{\mathrm{NV} \odot} & =\sum_{i} \gamma_{\mathrm{NV}} \gamma_{\odot}^{i} \frac{\mu_{0}}{4 \pi} \frac{\hbar^{2}}{r^{3}}\left[\mathbf{S}_{\mathbf{N V}} \cdot \mathbf{S}_{\odot}^{i}-\frac{3}{r^{3}}\left(\mathbf{S}_{\mathbf{N V}} \cdot \mathbf{r}\right)\left(\mathbf{S}_{\odot}^{i} \cdot \mathbf{r}\right)\right], \\
K_{\odot \odot} & =\sum_{i, j} \gamma_{\odot}^{i} \gamma_{\odot}^{j} \frac{\mu_{0}}{4 \pi} \frac{\hbar^{2}}{r^{3}}\left[\mathbf{S}_{\odot}^{i} \cdot \mathbf{S}_{\odot}^{j}-\frac{3}{r^{3}}\left(\mathbf{S}_{\odot}^{i} \cdot \mathbf{r}\right)\left(\mathbf{S}_{\odot}^{j} \cdot \mathbf{r}\right)\right] .
\end{aligned}
$$

In the subsequent analysis, the response of the system to this Hamiltonian will be solved numerically using a Lindblad master equation [47] to describe decoherence effects on the target and NV spins:

$$
\begin{aligned}
\dot{\rho} & =-\frac{l}{\hbar}\left[H_{\text {system }}, \rho\right]+L_{\text {total }}, \\
L_{\text {total }} & =L_{\mathrm{NV} T_{1}}+L_{\mathrm{NV} T_{2}}+L_{\odot T_{1}}+L_{\odot T_{2}},
\end{aligned}
$$

where $\rho$ is the density matrix of the system, $L_{\mathrm{NV} T_{1 / 2}}$ and $L_{\odot T_{1 / 2}}$ describe the loss of coherence of the NV spin and the target nuclear spins, respectively, due to $T_{1}$ and $T_{2}$ processes, as given by the following Lindblad terms:

$$
\begin{aligned}
L_{\mathrm{NV} T_{1}} & =\frac{1}{T_{1 \mathrm{NV}}}\left[l_{\mathrm{NV}} \rho l_{\mathrm{NV}}^{\dagger}-\frac{1}{2}\left(\rho l_{\mathrm{NV}} l_{\mathrm{NV}}^{\dagger}+l_{\mathrm{NV}} l_{\mathrm{NV}}^{\dagger} \rho\right)\right] \\
L_{\mathrm{NV} T_{2}} & =\frac{1}{T_{2 \mathrm{NV}}}\left[Z_{\mathrm{NV}} \rho Z_{\mathrm{NV}}^{\dagger}-\frac{1}{2}\left(\rho Z_{\mathrm{NV}} Z_{\mathrm{NV}}^{\dagger}+Z_{\mathrm{NV}} Z_{\mathrm{NV}}^{\dagger} \rho\right)\right], \\
L_{\odot T_{1}} & =\frac{1}{T_{1 \odot}} \sum_{i} l_{\odot}^{i} \rho l_{\odot}^{i \dagger}-\frac{1}{2}\left(\rho l_{\odot}^{i} l_{\odot}^{i \dagger}+l_{\odot}^{i} l_{\odot}^{i \dagger} \rho\right) \\
L_{\odot T_{2}} & =\frac{1}{T_{2 \odot}} \sum_{i} Z_{\odot}^{i} \rho Z_{\odot}^{i \dagger}-\frac{1}{2}\left(\rho Z_{\odot}^{i} Z_{\odot}^{i \dagger}+Z_{\odot}^{i} Z_{\odot}^{i \dagger} \rho\right)
\end{aligned}
$$

where $l_{\mathrm{NV} / \odot}$ is the lowering operator for the $\mathrm{NV}$ or target spin.

It is instructive to analytically obtain, using the secular approximation (given the large zero field splitting of the NV levels), the interaction strength $k(\mathbf{r})$ between a NV center and only one nuclear spin:

$$
k(\mathbf{r})=\gamma_{\mathrm{NV}} \gamma_{\odot} \hbar \frac{\mu_{0}}{4 \pi} \frac{1}{r^{3}}\left(1-\frac{3 r_{z}^{2}}{r^{2}}\right),
$$

where $r_{z}$ is the $z$ component of distance $r$ between the NV center and target spin.

Figure 2(c) shows a lobelike contour of the function $k(\boldsymbol{r})$. As the shells of equivalent interaction strength are not radially symmetric, it is convenient to define the distance between the NV center and the target $r_{\max }$ as the $z$-axis intercept of the $k(\boldsymbol{r})$ shell on which the target is located, as depicted in Fig. 2(c). Therefore, $r_{\max }$ defines a maximal physical distance between the NV center and a target for a particular interaction strength. Considering the examples analyzed in this paper, the relationship between $r_{\max }$ and the interaction strength for one proton spin becomes

$$
k\left(r_{\max }\right) \approx \frac{1 \mathrm{MHz} \times \mathrm{nm}^{3}}{r_{\max }^{3}} .
$$

\section{B. Comparison with liquid and solid state NMR}

Traditionally, NMR techniques are classified by their environment being either liquid or solid state. These two cases produce fundamentally different NMR spectra [48-50] and require further insight in light of applications of our method.

A liquid NMR spectrum is dominated by isotropic $J$ coupling and chemical shift while any other anisotropic interactions, including dipole-dipole (d-d) coupling, typically average out due to constant Brownian rotation with respect to the background magnetic field. Thus, molecules in a solution possess a relatively simple spectrum with sharp resonant peaks, particularly at high background fields. Consequently, the coherence times of proton spins in liquids tend to be very long. Being highly localized, a NV center would, in this case, require a high concentration of target molecules for a successful detection process.

A solid state NMR spectrum is strongly dominated by a dipole-dipole interaction, while the influence of $J$ coupling and chemical shifts depend on the molecule and the background magnetic field. It is the dominant nature of the anisotropic interactions that makes the solid state NMR spectrum relatively broadened, yet rich with spatial information. In a powdered form, an ensemble of possible molecular 
orientations creates wide regions of resonant frequencies instead of single peaks. Single crystal samples have more defined peaks dependent on the lattice orientation, however they are not a suitable biomolecular platform. A number of techniques have been developed to eliminate the anisotropic coupling in noncrystalline samples, which require macroscopic equipment and bulk samples [49]. However, such techniques have no capacity to extract the spatial information contained in the anisotropic effects.

Molecules bonded to the surface of diamond are effectively stationary and do not exhibit anisotropic broadening in the present context. If the background magnetic field is constant over the diamond surface, the orientation of each surface bonded molecule with respect to the background field will be uniform. These requirements are not difficult to achieve locally, as the detection area directly above the NV center is of a nanometer scale. Furthermore, when the surface density of the molecules is not large (e.g., $<1 / \mathrm{nm}^{2}$ ), the nuclear coherence times are relatively long $(>1 \mathrm{~ms})$. Additional information about the diamond surface chemistry and its potential for biochemical applications can be found in Ref. [51]. However, the number of molecules bonded to the microscopic diamond surface is generally too small for conventional NMR characterization. In the following examples we focus on the case where both the NV center and target molecule densities are such that one target molecule is in the detection range of one NV center preventing orientation broadening of the NMR target spectrum. Isolated single NV centers are routinely engineered via chemical growth techniques and $\mathrm{N}$ ion implantation [34,35,52-54].

\section{RESULTS AND DISCUSSION}

In this section we apply our method to the concrete examples of three chemical groups outlined above. First, the NMR spectrum of each group is characterized. Second, the molecular detection based on NMR peak locations is discussed in the context of its full parameter space.

The target molecule NMR spectrum characterization is as follows. To obtain a reference signal, a NV center is characterized to a high precision without a rf field present using the preferred control sequence. We demonstrate this method using a spin-echo sequence. Assuming the background magnetic field is aligned with the NV spin quantization axis, it is sufficient to choose a single sensitive time point $t_{\mathrm{p}}$ on the signal envelope as illustrated in Fig. 2(b). A sweep over the $\mathrm{rf}$ frequency $\omega$ is then performed, while the NV center is simultaneously observed at the point $t_{\mathrm{p}}$ as per the chosen control sequence. When the rf field becomes resonant with the target nuclear transition, the signal from the NV center will show an associated increase.

\section{A. The NMR spectrum}

Figure 3 shows the numerically simulated NMR spectra of three single chemical groups, characterized using a NV center located $r_{\max }=5 \mathrm{~nm}$ below the surface of an ultrapure bulk diamond. The spin-echo sequence was applied to the NV center while the resonant rf field was applied continuously. The control pulses for the NV center were assumed to be perfect, as the detuning of the NV electronic spin due to the nuclear spins of the molecule is relatively small for the considered distances. The initial spin state of the system was a mixed state, reflecting a high temperature environment; and the background field was taken to be $B_{0}=0.01 \mathrm{~T}$, as it is easily reproduced experimentally compared to many other NMR applications requiring a significantly stronger fields $(\geqslant 1 \mathrm{~T})$. For a low background magnetic field case, dipole-dipole interaction between the target species nuclei is the most dominant as compared with other internuclear interactions. The results demonstrate a sufficient level of sensitivity to perform single-molecule NMR spectroscopy, while multiple targets contribute to the signal strength in a simple manner. However, when using the higher order sequences (CPMG or Uhrig) to further improve the sensitivity, a trade-off may be necessary as the Rabi frequency of the target spin has to be synchronized with the control pulses of the NV center sequence [21,24,57]. This requires a stronger rf field which is in turn a limiting factor to spectral resolution either because of broadening or experimental constraints.

The spectral analysis (Fig. 3) was carried out for a particular case in which the NV center was aligned along the $\langle 111\rangle$ crystallographic axis. In this case, the background magnetic field and NV axis are aligned normal to the diamond surface. Different orientations of the NV center produce no fundamental change to the measurement procedure, but the orientation change results in two characteristic effects. As the background magnetic field and the NV axis are always considered aligned, a change in the orientation of the NV center and consequently the background magnetic field would change the target spectrum, as it is produced by anisotropic dipole-dipole nuclear spin interactions. Furthermore, changing the NV quantization axis modifies the coupling strength [see Eq. (5)] between the target and the NV center, thereby impacting the sensitivity.

\section{B. Molecular detection and optimization}

Once the NMR spectrum of the target molecules has been characterized, the NV center can be used as a selective single-molecule detector. The proposed method is as follows. A number of resonant frequencies that identify particular species are selected. The signal is then screened for only those particular frequencies. If the targeted species is present the signal is observed. There are a number of approaches to analyzing the change in population, depending on the shape of the signal when no rf field is present. In line with the previous description, it is sufficient to consider a single temporal point $t_{\mathrm{p}}$. For each individual targeted transition, a resonant field of strength $B_{\mathrm{NMR}}$ is applied while the NV spin population is observed for a control sequence of length $t_{\mathrm{p}}$. Detection of a target species with this particular transition is achieved if the signal $\Delta S$ is observed. As the signal is detected optically, its uncertainty is shot noise limited. Confirmation of the presence of the target can be defined as the moment when the signal to noise ratio exceeds unity:

$$
\Delta S=S\left(B_{\mathrm{NMR}}=0\right)-S\left(B_{\mathrm{NMR}} \neq 0\right) \geqslant \frac{1}{C \sqrt{N}}
$$




\section{Single molecule species detection parameters}
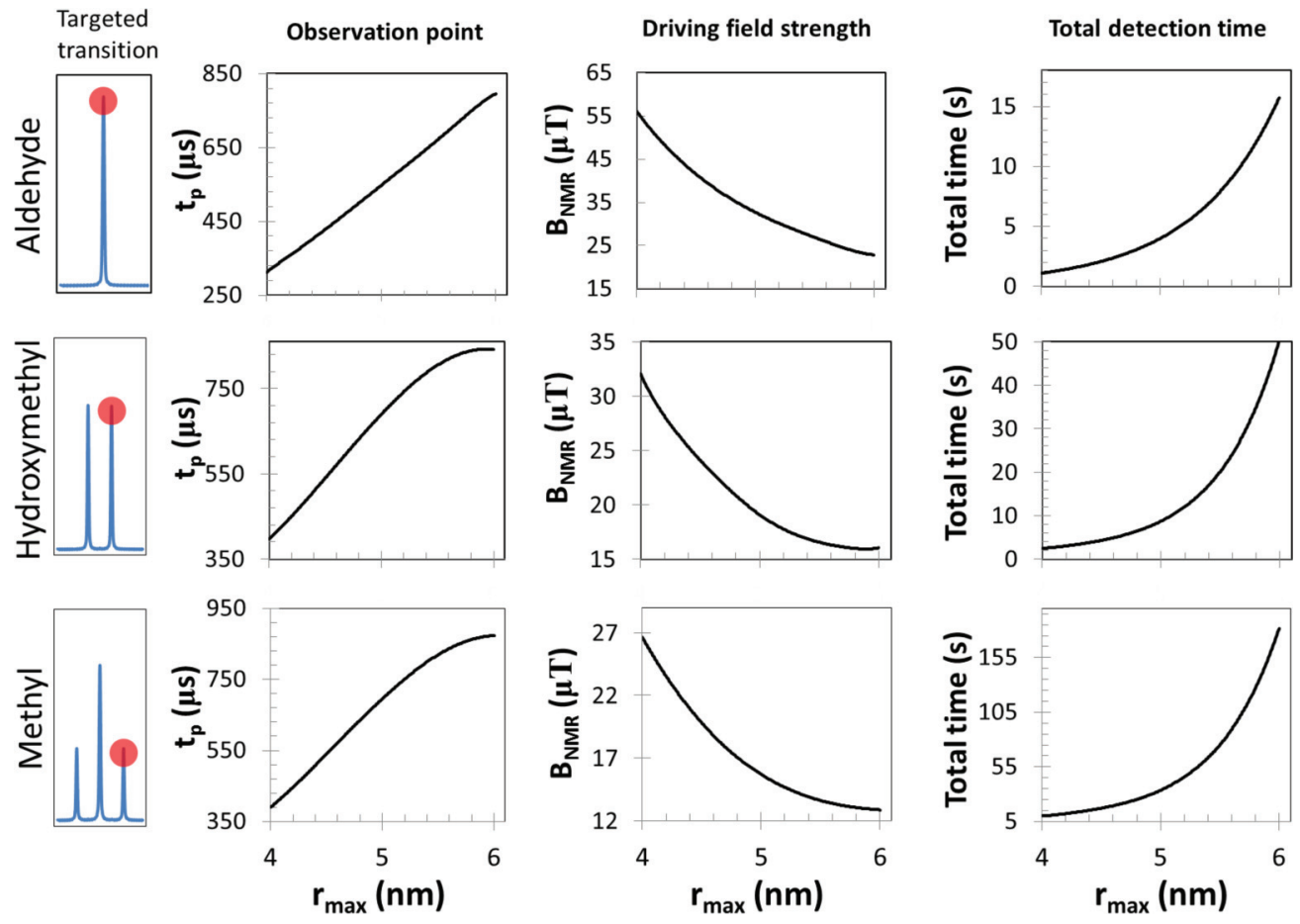

FIG. 4. (Color online) Optimized detection time as a function of distance for a highlighted proton transition. This analysis demonstrates that detection time scales of an order of seconds are achievable using continually driven targeted species and a spin-echo measurement sequence for the NV center. The optimal parameters for such detection are also shown corresponding to the rf field strength $B_{\mathrm{NMR}}$ and the time point $t_{\mathrm{p}}$ at which the NV centers signal is monitored.

where $N$ is the number of measurements, and $C$ is a parameter describing effects such as signal contrast and photon collection efficiency [21]. This also facilitates the quantification of this method's temporal resolution, as the total time to detect a targeted transition becomes $T=N t_{\mathrm{p}}$.

The magnitude of the signal depends on the interaction strength $k(\boldsymbol{r})$ between the target nucleus and the NV center, which is distance dependent, thereby dictating the spatial resolution of the method. The signal strength is dependent on many factors: the target distance $r_{\max }$, the time point $t_{\mathrm{p}}$, the strength of the rf field $B_{\mathrm{NMR}}$, the coherence times of the NV center/target species, and the number of target molecules in the vicinity of the NV center. It also depends on the type of

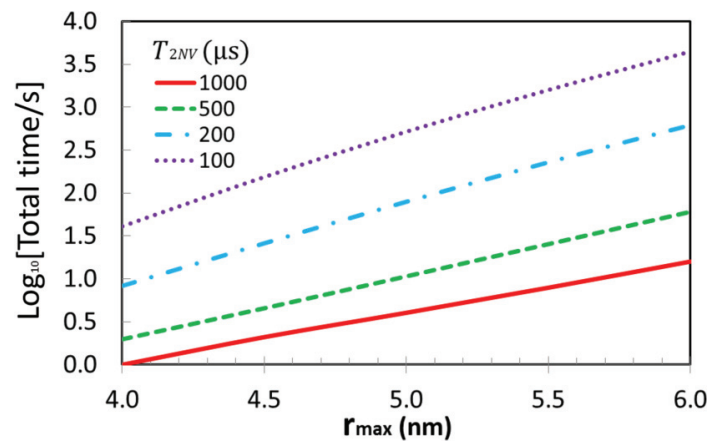

FIG. 5. (Color online) Optimized detection time for sensing a single proton over a range of distances $\left(r_{\max }\right)$ from the NV center for several transverse coherence times of the $\mathrm{NV}$ center. the targeted transition and the number of nuclei it involves. To explore the full potential of the detection method, we investigate the relationship between these parameters. Figure 4 shows the complexity of the system's parameter space. Three different transitions have been considered under a spin-echo sequence for the $\mathrm{NV}$ center. For each, the relationship between $r_{\mathrm{max}}, t_{\mathrm{p}}$, and $B_{\mathrm{NMR}}$ was analyzed, including an optimization for the temporal resolution. The parameters chosen for the simulations (Figs. 3 and 4) reflect presently achievable experimental conditions as follows: $B_{0}=0.01 \mathrm{~T}, C=0.05, T_{1 \mathrm{NV}}=5 \mathrm{~ms}$, $T_{2 \mathrm{NV}}=1 \mathrm{~ms}[12,20,45,58,59], T_{1 \odot}=10 \mathrm{~ms}, T_{2 \odot}=1 \mathrm{~ms}$. A significant limiting factor in the present NV-based detection is the NV spins's transverse coherence time $T_{2 \mathrm{NV}}$, which, for shallow NV centers, is strongly influenced by the diamond surface conditions. Figure 5 shows the trend of the total detection time associated with decrease in $T_{2 \mathrm{NV}}$ for a single proton target over a rage of distances. These results indicate that the detection method may be used under a range of accessible coherence conditions, however, there is a decrease in temporal resolution with the loss of coherence.

\section{CONCLUSION}

There is a large scope for the application of single-molecule detection using the NV center. One example is in the detection of molecular bonding processes to the diamond surface, or monitoring an intermediate (antenna) molecule whose NMR spectrum depends on a particular external factor. The antenna molecule my change its structure due to a range of factors 
leading to a change in its NMR spectrum, for example, interaction with another molecule, a change in $p \mathrm{H}$ value of the solution, or a change in the surrounding electric field or local temperature. The examples considered in this paper explicitly considered molecules covalently bonded to the surface of the diamond, however, it is worth noting that the method can be applied to any type of bonding that makes the molecules motionless for time scales of the order of the temporal resolution of the detection.

An important feature that sets the method outlined in this paper apart from other NV based detection schemes is that it allows for simultaneous observation of more than one target transition. The basic principle consists of applying a number of rf fields, while barcoding each applied frequency with different field strength, insuring that the signal associated with each frequency appears at distinct time points $t_{\mathrm{p}}$ on the signal envelope. Thus, the presence of any particular targeted transition is directly observed from the signal graph. Alternatively, if the increase in signal is desired with no need for individual transition identification, strengths of each rf field can be tuned to produce coinciding signal peaks at the same $t_{\mathrm{p}}$, inducing a collective contributing to the signal strength. Additional increases in sensitivity would be achieved through improvements of such experimental parameters as the photon collection efficiency and NV spin coherence times.

We have investigated the application of NV sensing to single-molecule NMR detection through a direct and selective driving of the target molecular nuclear transitions. The analysis was carried out for continuous rf driving of a targeted transition, while the signal was obtained through the application of a spin-echo sequence on the NV center. Our theoretical analysis indicates that detection is achievable with temporal resolution in the range of seconds and spectral resolution sufficiently high to allow determination of the molecular species.

\section{ACKNOWLEDGMENTS}

The authors would like to thank Alastair Stacey and Kane O'Donnell for useful discussions. This work was supported by the Australian Research Council under the Centre of Excellence scheme (project number CE110001027).
[1] P. P. Borbat, A. J. Costa-Filho, K. A. Earle, J. K. Moscicki, and J. H. Freed, Science 291, 266 (2001).

[2] A. Mittermaier and L. E. Kay, Science 312, 224 (2006).

[3] D. Rugar, R. Budakian, H. J. Mamin, and B. W. Chui, Nature (London) 430, 329 (2004).

[4] A. Vinante, G. Wijts, O. Usenko, L. Schinkelshoek, and T. H. Oosterkamp, Nat. Commun. 2, 572 (2011).

[5] H. J. Mamin, R. Budakian, B. W. Chui, and D. Rugar, Phys. Rev. B 72, 024413 (2005).

[6] M. W. Doherty, N. B. Manson, P. Delaney, F. Jelezko, J. Wrachtrup, and L. C. L. Hollenberg, Phys. Rep. 528, 1 (2013).

[7] J. Wrachtrup, C. von Borczyskowski, J. Bernard, M. Orritt, and R. Brown, Nature (London) 363, 244 (1993).

[8] A. Gruber, A. Drbenstedt, C. Tietz, L. Fleury, J. Wrachtrup, and C. v. Borczyskowski, Science 276, 2012 (1997).

[9] F. Jelezko and J. Wrachtrup, J. Phys.: Condens. Matter 16, R1089 (2004).

[10] J. Harrison, M. J. Sellars, and N. B. Manson, J. Lumin. 107, 245 (2004).

[11] N. B. Manson, J. P. Harrison, and M. J. Sellars, Phys. Rev. B 74, 104303 (2006).

[12] G. Balasubramanian, P. Neumann, D. Twitchen, M. Markham, R. Kolesov, N. Mizuochi, J. Isoya, J. Achard, J. Beck, J. Tissler et al., Nat. Mater. 8, 383 (2009).

[13] Y.-R. Chang, H.-Y. Lee, K. Chen, C.-C. Chang, D.-S. Tsai, C.-C. Fu, T.-S. Lim, Y.-K. Tzeng, C.-Y. Fang, C.-C. Han et al., Nat. Nano 3, 284 (2008).

[14] F. Neugart, A. Zappe, F. Jelezko, C. Tietz, J. P. Boudou, A. Krueger, and J. Wrachtrup, Nano Lett. 7, 3588 (2007).

[15] L. P. McGuinness, Y. Yan, A. Stacey, D. A. Simpson, L. T. Hall, D. Maclaurin, S. Prawer, P. Mulvaney, J. Wrachtrup, F. Caruso et al., Nat. Nano 6, 358 (2011).

[16] M. V. G. Dutt, L. Childress, L. Jiang, E. Togan, J. Maze, F. Jelezko, A. S. Zibrov, P. R. Hemmer, and M. D. Lukin, Science 316, 1312 (2007).
[17] T. D. Ladd, F. Jelezko, R. Laflamme, Y. Nakamura, C. Monroe, and J. L. O'Brien, Nature (London) 464, 45 (2010).

[18] A. Beveratos, R. Brouri, T. Gacoin, A. Villing, J.-P. Poizat, and P. Grangier, Phys. Rev. Lett. 89, 187901 (2002).

[19] C. Kurtsiefer, S. Mayer, P. Zarda, and H. Weinfurter, Phys. Rev. Lett. 85, 290 (2000).

[20] G. Balasubramanian, I. Y. Chan, R. Kolesov, M. Al-Hmoud, J. Tisler, C. Shin, C. Kim, A. Wojcik, P. R. Hemmer, A. Krueger et al., Nature (London) 455, 648 (2008).

[21] J. M. Taylor, P. Cappellaro, L. Childress, L. Jiang, D. Budker, P. R. Hemmer, A. Yacoby, R. Walsworth, and M. D. Lukin, Nat. Phys. 7, 270 (2011).

[22] S. Steinert, F. Dolde, P. Neumann, A. Aird, B. Naydenov, G. Balasubramanian, F. Jelezko, and J. Wrachtrup, Rev. Sci. Instrum. 81, 043705 (2010).

[23] J. H. Cole and L. C. L. Hollenberg, Nanotechnology 20, 495401 (2009).

[24] L. T. Hall, J. H. Cole, C. D. Hill, and L. C. L. Hollenberg, Phys. Rev. Lett. 103, 220802 (2009).

[25] L. T. Hall, C. D. Hill, J. H. Cole, B. Stdler, F. Caruso, P. Mulvaney, J. Wrachtrup, and L. C. L. Hollenberg, Proc. Natl. Acad. Sci. USA 107, 18777 (2010).

[26] C. A. Meriles, L. Jiang, G. Goldstein, J. S. Hodges, J. Maze, M. D. Lukin, and P. Cappellaro, J. Chem. Phys. 133, 124105 (2010).

[27] B. Grotz, J. Beck, P. Neumann, B. Naydenov, R. Reuter, F. Reinhard, F. Jelezko, J. Wrachtrup, D. Schweinfurth, B. Sarkar et al., New J. Phys. 13, 055004 (2011).

[28] L. P. McGuinness, L. T. Hall, A. Stacey, D. A. Simpson, C. D. Hill, J. H. Cole, K. Ganesan, B. C. Gibson, S. Prawer, P. Mulvaney et al., New J. Phys. 15, 073042 (2013).

[29] H. J. Mamin, M. H. Sherwood, and D. Rugar, Phys. Rev. B 86, 195422 (2012).

[30] M. S. Grinolds, S. Hong, P. Maletinsky, L. Luan, M. D. Lukin, R. L. Walsworth, and A. Yacoby, Nat. Phys. 9, 215 (2013). 
[31] S. Steinert, F. Ziem, L. Hall, A. Zappe, M. Schweikert, A. Aird, G. Balasubramanian, L. Hollenberg, and J. Wrachtrup, arXiv:1211.3242.

[32] S. Kaufmann, D. A. Simpson, L. T. Hall, V. Perunicic, P. Senn, S. Steinert, L. P. McGuinness, B. C. Johnson, T. Ohshima, F. Caruso et al., Proc. Natl. Acad. Sci. USA 110, 10894 (2013).

[33] A. Ermakova, G. Pramanik, J.-M. Cai, G. Algara-Siller, U. Kaiser, T. Weil, Y.-K. Tzeng, H.-C. Chang, L. P. McGuinness, M. B. Plenio et al., Nano Lett. 13, 3305 (2013).

[34] T. Staudacher, F. Shi, S. Pezzagna, J. Meijer, J. Du, C. A. Meriles, F. Reinhard, and J. Wrachtrup, Science 339, 561 (2013).

[35] H. J. Mamin, M. Kim, M. H. Sherwood, C. T. Rettner, K. Ohno, D. D. Awschalom, and D. Rugar, Science 339, 557 (2013).

[36] N. Zhao, J. Honert, B. Schmid, J. Isoya, M. Markham, D. Twitchen, F. Jelezko, R.-B. Liu, H. Fedder, and J. Wrachtrup, arXiv:1204.6513.

[37] S. Kolkowitz, Q. P. Unterreithmeier, S. D. Bennett, and M. D. Lukin, Phys. Rev. Lett. 109, 137601 (2012).

[38] N. Zhao, J.-L. Hu, S.-W. Ho, J. T. K. Wan, and R. B. Liu, Nat. Nano 6, 242 (2011).

[39] J. Cai, F. Jelezko, M. B. Plenio, and A. Retzker, New J. Phys. 15, 013020 (2013).

[40] E. L. Hahn, Phys. Rev. 80, 580 (1950).

[41] S. Meiboom and D. Gill, Rev. Sci. Instrum 29, 688 (1958).

[42] G. S. Uhrig, Phys. Rev. Lett. 98, 100504 (2007).

[43] J. M. Taylor, P. Cappellaro, L. Childress, L. Jiang, D. Budker, P. R. Hemmer, A. Yacoby, R. Walsworth, and M. D. Lukin, Nat. Phys. 4, 810 (2008).

[44] L. T. Hall, C. D. Hill, J. H. Cole, and L. C. L. Hollenberg, Phys. Rev. B 82, 045208 (2010).

[45] B. Naydenov, F. Dolde, L. T. Hall, C. Shin, H. Fedder, L. C. L. Hollenberg, F. Jelezko, and J. Wrachtrup, Phys. Rev. B 83, 081201 (2011).
[46] G. de Lange, D. Ristè, V. V. Dobrovitski, and R. Hanson, Phys. Rev. Lett. 106, 080802 (2011).

[47] H. Breuer and F. Petrucci, The Theory of Open Quantum Systems (Oxford University Press, Oxford, 2002).

[48] L. Schröder, C. Schmitz, and P. Bachert, J. Magn. Reson. 171, 213 (2004).

[49] I. Schnell, Curr. Anal. Chem. 1, 3 (2005).

[50] D. Cremer and J. Grafenstein, Phys. Chem. Chem. Phys. 9, 2791 (2007).

[51] A. Krueger and D. Lang, Adv. Funct. Mater. 22, 890 (2012).

[52] A. Stacey, D. A. Simpson, T. J. Karle, B. C. Gibson, V. M. Acosta, Z. Huang, K. M. C. Fu, C. Santori, R. G. Beausoleil, L. P. McGuinness et al., Adv. Mater. 24, 3333 (2012).

[53] T. Ishikawa, K.-M. C. Fu, C. Santori, V. M. Acosta, R. G Beausoleil, H. Watanabe, S. Shikata, and K. M. Itoh, Nano Lett. 12, 2083 (2012).

[54] K. D. Jahnke, B. Naydenov, T. Teraji, S. Koizumi, T. Umeda, J. Isoya, and F. Jelezko, Appl. Phys. Lett. 101, 012405 (2012).

[55] R. Martín, M. lvaro, J. R. Herance, and H. García, ACS Nano 4, 65 (2010).

[56] N. Tokuda, D. Takeuchi, S.-G. Ri, H. Umezawa, K. Yamabe, H. Okushi, and S. Yamasaki, Diam. Relat. Mater. 18, 213 (2009).

[57] R. Hanson, F. M. Mendoza, R. J. Epstein, and D. D. Awschalom, Phys. Rev. Lett. 97, 087601 (2006).

[58] J. R. Maze, P. L. Stanwix, J. S. Hodges, S. Hong, J. M. Taylor, P. Cappellaro, L. Jiang, M. V. G. Dutt, E. Togan, A. S. Zibrov et al., Nature (London) 455, 644 (2008).

[59] L. Childress, M. V. Gurudev Dutt, J. M. Taylor, A. S. Zibrov, F. Jelezko, J. Wrachtrup, P. R. Hemmer, and M. D. Lukin, Science 314, 281 (2006). 